\title{
An Investigation into the Effect Of Stochastic Annual Rainfall on Crop Yields in South Western Australia
}

\author{
Yunous Vagh
}

\begin{abstract}
In this research, the methodology of action research dynamics and a case study using both qualitative and quantitative methods was employed for the analysis of geographic data in an agricultural context. The geographic data was made up of land use profiles that were juxtaposed with previously captured rainfall data from fixed weather stations in Australia which was interpolated using ordinary krigeing to fit a grid surface. The resultant stochastic annual rainfall profiles for a selected study area within the South West Agricultural region of Western Australia were used to identify areas of high crop production. The areas within the study area were spatially scaled to individual shires. The rainfall was sampled for the years 2002, 2003, 2005 as a mix of low and high rainfall and high production attributes. The patterns suggested that crop production was closely linked to the annual rainfall for some shires, with location being of significance at other shires.
\end{abstract}

Index Terms-GIS, spatial scaling, data mining, annual stochastic rainfall, crop yields.

\section{INTRODUCTION}

The aim of this work was to find a relationship between rainfall, land location (shire) and crop production and in order to justify agricultural land-use, and to also possibly predict the crop production yield at certain locations within the agricultural region, given the rainfall. As far back as in 1979, Anderson singled out low rainfall as the factor behind most of the adversities of the agricultural sector [1]. In fact in the past for example, low rainfall has been attributed to be the cause of large drops in production yield as much as $18 \%$ in 1983 [2]. According to Olesin and Bindi (2002) the factors of radiation, temperature and rainfall all affect yield to some degree with rainfall especially affecting the growth and production of the plant [3]. Variability in rainfall from year to year is closely intertwined with crop yields [4] and although a great deal of the variability of Australian rainfall may be related to the either the El Nino - Southern Oscillation (ENSO) or sea surface temperature (SST) phenomena [5], previous studies have shown the predictions not to be altogether accurate as pointed out by McBride and Nicholls (1983), Pittock (1984) [5, 6] as well as by Nicholls (1985) [7] Furthermore, down-upscaling was not carried out in this research as the yield was not correlated with an oceanic index such as ENSO. This study however, considered the linear relationship between crop yield and rainfall similar to studies such as Challinor et.al (2003) [8] where there was an established physical basis such as a spatial scale upon which

Manuscript received March 16, 2012; revised April 23, 2012.

Y. Vagh is with Edith Cowan University, Perth, Western Australia (e-mail: vvagh@ecu.edu.au) the variables operated. Spatial scales are important in that the scale related results are specific to the related group or agency. For example, the national scale may be used by governments to determine their economic strategy from food reserves [9], while results from smaller scale relationships, being used to detect food shortages and associated mitigation possibilities [10], and for seasonal forecasting by farmers at the farm level [11]. This study differs from Challinor's in that it will investigate the correlations between these factors on a spatial scale that is somewhere between the farm level (small) and the whole agricultural region level (large), and that is the shire level (medium). This was due mainly to the availability of crop yield data on this level as well as the way in which the Western Australian system operates. In order to limit the analysis for the purposes of focus and scope as well as to highlight the relationships under rainfall and yield variability, three specific years were chosen. These were the years 2002 (low rainfall), 2003 (high yield) and 2005 (high rainfall).

\section{RELATED WORK}

The use of observed relationships such as rainfall to predict crop yield has been undertaken by Parthasarathy et al (1992) through an empirical model [12]. Interactions between input variables such as rainfall and output variables such as crop yield have been shown to be important $[13,14]$. In particular, their relevance was emphasized at critical phenological growth stages [15]. A relational analysis such as this, needs to be based on a model. As such there are two approaches for developing models and these are the process based crop models (CM) for the establishment of non-linear relationships between weather variables and crop yield, and general circular models (GCM) of the coupling of the ocean and the atmosphere. The use of GCMs for prediction at a seasonal lead-time have been shown to suffer from the problem of simulation of too many low intensity rainfall instances within each grid cell [16]. As short term weather forecasting rather than long-term climate forecasting is important [8], this study is therefore more suited to the CM approach. Previous researchers have concluded that the complexity of a model can be based on the level of detail of the analysis [17] or it can be less detailed with only estimations of moisture content [18]. Other approaches have been the use of the normalized difference vegetative index (NDVI) for grouping homogeneous regions to establish the scale [19]. There have also been approaches to crop modelling using the derivation of a probability distribution function (PDF) for the assessment of quantifying the risks and benefits of making weather based decisions [3]. Although, according to Sivakumar (2006), notwithstanding 
that considerable improvements in understanding and predicting climate variability have been made, the need to further develop understanding and refine tools is ever increasing [20], especially because the atmosphere is intrinsically a chaotic system.

\section{MATERIALS AND METHODS}

The research methodology was developed after experimentation with a number of software tools that represented an admixture of extraction, pre-processing, analysis, data mining and visualization of climate data sourced from the Department of Agriculture and Food of Western Australia (DAFWA). It involved both a qualitative (visual inspection) and a quantitative (data mining) research method. The data was made up of geographical land-use profiles, rainfall data and crop production data. The study area was a rectangular grid of 104328100 hectare cells, stretching from Busselton in the west, to Esperance in the east for the South Western Agricultural region. The ERMapper, ARCgis and GeoMedia software packages were used to create the study area, and the various GIS profiles. In addition, all the datasets were fitted specifically to the extraction region of the selected study area. Each of the shires within the study area was made up of a number of the 100ha grid cells whose coordinates were matched up with the interpolated rainfall data.

\section{A. Rainfall Data and Interpolation}

Historical rainfall and climate data existed only at sparsely located weather stations within the study area. In order to overcome the limitation of a sparse rainfall dataset, a process of interpolation was carried out resulting in stochastic rainfall data points at each cell of the study area grid. The interpolation was carried out in the Revolution R script for each of the 12 months for the years 2001 to 2010 and fitted onto the study area grid surface. The interpolation was done at a high resolution of $1000 \mathrm{~m}$ by $1000 \mathrm{~m}$. Uncertainty due to spatial elevation bias was minimal due to the weather stations being a homogenous group. As analysis was to be only be done for the years 2002 (low rainfall), 2003 (high yield) and 2005 (high rainfall) rainfall years, only the rainfall data for these years were extracted. The coordinates of the extracted interpolated rainfall data were matched up with the production data for the same three selected years.

\section{B. Production Data}

The production data only existed at an annual level for each shire within the study area. Consequently, the rainfall data had to be aggregated to an annual level using an R Script to match the annual crop yield data for the years 2002, 2003 and 2004 only as part of the normalization process. A process of data reduction was performed on the full study area dataset by only selecting land uses reserved for cropping and cereals. Next, the dataset was sorted into shire order and the rainfall for each of the cells within each shire was scaled by the number of cells within each shire to produce a single line for the shire, average annual rainfall, and annual wheat crop yield for each shire. This dataset was then used for the subsequent analyses using rainfall and wheat yield.

\section{EXPERIMENTAL RESULTS AND ANALYSIS}

There were a number of aspects to the data handling and analyses. They included the pre-processing and metrics, the analysis of the rainfall and the analysis of the wheat yields which formed the macroscopic phase. In addition, there was the data mining (DM) analysis of the wheat crop yields which formed the microscopic phase.

\section{A. Pre-Processing and Metrics used}

The final analysis was carried out on a shire level for the two attributes of stochastic average annual rainfall and actual crop yield per shire. The 23 crop production shires were mapped separately in QuantumGis to visualise the size and location of each shire as shown in Fig. 1. The annual crop production data was calculated in tonnes per hectare, in order to account for the different shire sizes.

The visual inspection of the graphs required a uniform method of evaluation. This was in the form of a baseline metric of classifying the shires into rainfall categories of very high rainfall (VHR) of over $600 \mathrm{~mm}$ per annum, high rainfall (HR) of 500-600mm per annum and low rainfall (LR) of less than $500 \mathrm{~mm}$ per annum. The shires were also classified as high yield (HY) of over 45,000 tonnes per shire and low yield (LY) of less than 45,000 tonnes per shire per annum based on the wheat yield for 2003. Accordingly, the HY shires were Dumbleyung, Esperance, Gnowangerup, Jerramungup, Katanning, Kent, Kulin, Lake Grace, Ravensthorpe and Wickepin. The LY shires were Boddington, Boyup Brook, Broomehill, Bridgetown-Greenbushes, Cranbrook, Kojonup, Manjimup, Narrogin, Tambellup, Wagin, West Arthur, Williams and Woodanilling. All the 23 shires are shown in Fig. 1.

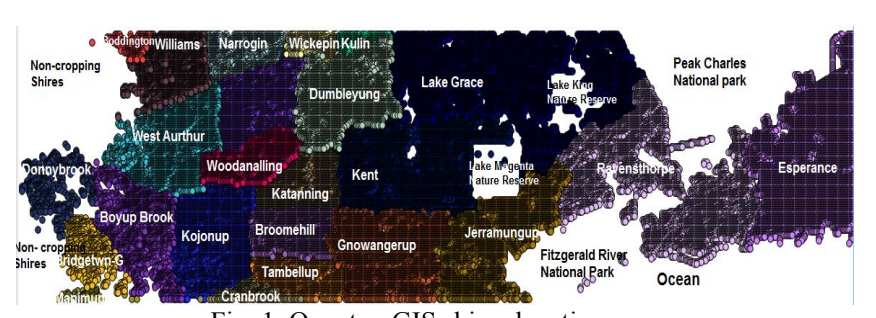

Fig. 1 QuantumGIS shires location map

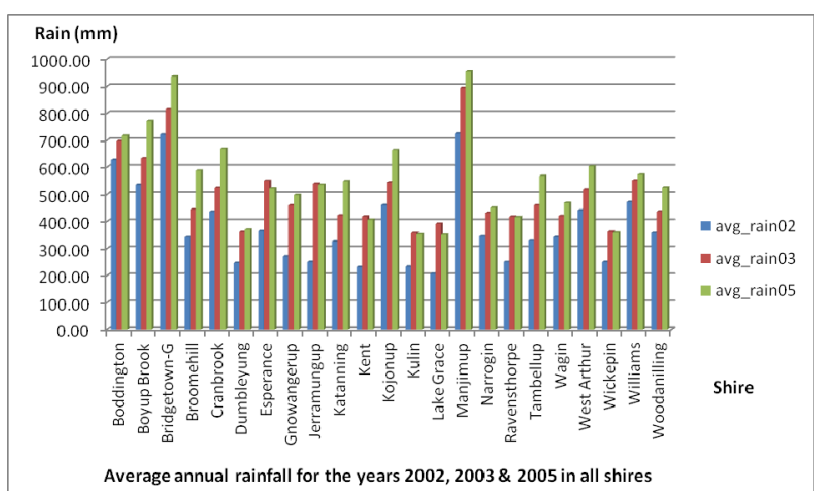

Fig. 2. Average annual rainfall for 2002, 2003 \& 2005

\section{B. Analysis of the Rainfall}

The analyses that followed involved a process of examination from macroscopic inspection to microscopic scrutiny. The selected three year rainfall graphs were first plotted in order to establish the rainfall trend across the three selected years of the dry 2002 year, the productive 2003 year 
and the wet 2005 year. The rainfall step showed the stochastic average annual rainfall for the three selected years of 2002, 2003 and 2005 in Fig. 2. These were the dry, productive and wet years respectively. The rainfall variation over the three years did reflect and confirm the overall classification of dry and wet years where the rainfall increased progressively from 2002 to 2005 . The variation was also clearly visible across the range of the different shires where the VHR shires were Boddington, Boyup Brook, Bridgetown-Greenbushes and Manjimup with the remaining HR shires with a overall high rainfall of $500 \mathrm{~mm}$ per annum for the three years. The average annual rainfall in the HY shires was generally lower than for the LY shires across the selected years and was recognisable in the peaks of Boddington, Boyup Brook, Bridgetown-Greenbushes and Manjimup as per Fig. 2, with the biggest variation being over $500 \mathrm{~mm}$ of rainfall between the shires of Manjimup and Lake Grace.

\section{Analysis of the Wheat crop Yields}

The analysis of the individual crop yield across the selected years involved examining the wheat crop yields for the years 2002, 2003 and 2005 as shown in Figure 3. The overall 2003 wheat crop yield was higher than for the year 2005. The LY shires of Boyup Brook, Kojonup and West Arthur produced the highest crop yields between 4-5 tonnes/hectare for the year 2003. Bridgetown-Greenbushes had zero yield due to no areas sown, and as a consequence it was eliminated from the ensuing analyses. The overall wheat crop yield trend was higher for the year 2003 and it defied the increased rainfall trend from 2003 to 2005. Only the LY shires of Boddington and Narrogin, and the HY shire of Ravensthorpe matched the trend with higher 2005 wheat crop yields.

\section{Correlation Analysis of the Wheat Crop Yields}

The next step was the in-depth scrutiny of the microscopic phase which involved examining the wheat crop yield for patterns and prediction possibility. This was done through a process of aggregation of the wheat crop yields for the selected three years in order to determine the time sequence as well as for prediction of crop yields using data mining. Standardization was performed on the two differing scale attributes of rainfall and wheat in order to facilitate a simultaneous sequence chart plot in SPSS as shown in Fig. 4.

The visual comparison of the graph enabled a year by year comparative analysis which showed the correlation between the average annual stochastic rainfall and the wheat yield across the three selected years. The overall repetitive cycle for the three years was visible for both the lines. It also displayed the increased rainfall trend from 2002 and 2003 to 2005. In addition the crop yield across the three years was also mirrored, showing the increased crop yields over the relevant shires. Furthermore, the negative impacts on wheat crop yield were also demonstrated for the LY shire of Narrogin where the rainfall patterns were evident. The correlation coefficient for wheat yield and annual rainfall in the HY shires for the dry year 2002 was $0.49,0.32$ for the productive year, and 0.45 for the wet year 2005. The correlation was positive for the year 2002 and improved progressively for the years 2003 and 2005. The overall correlation coefficient for the three years taken together was 0.75 for the HY shires. On the other hand, the correlation coefficient for the LY shires after the shire of Manjimup was excluded as an outlier, was 0.32 for the dry year 2002, -0.04 for the productive year 2003 and 0.35 for the wet year 2005 . The overall correlation coefficient was 0.25 for the three years taken together. This indicted that the overall positive correlation for the HY shires was better than the for the LY shires. It also indicated that the LY shires tended to be very slightly negative with a correlation of -0.04 for the year 2003 . This suggested that an increase in rainfall would invariably result in an increase in crop yield across the selected shires, but the increase in wheat yield diminishes as the rainfall increases in some shires.

As an extension of this activity the standardised data of the 22 wheat production shires was separated into the HY shires and the LY shires. These were then plotted on a graph for further clarification of the correlation.

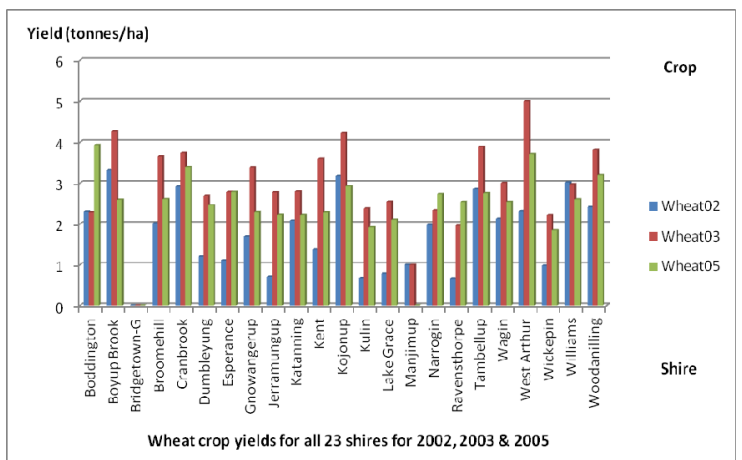

Fig. 3. Wheat crop yield for the years 2002, 2003 \& 2005

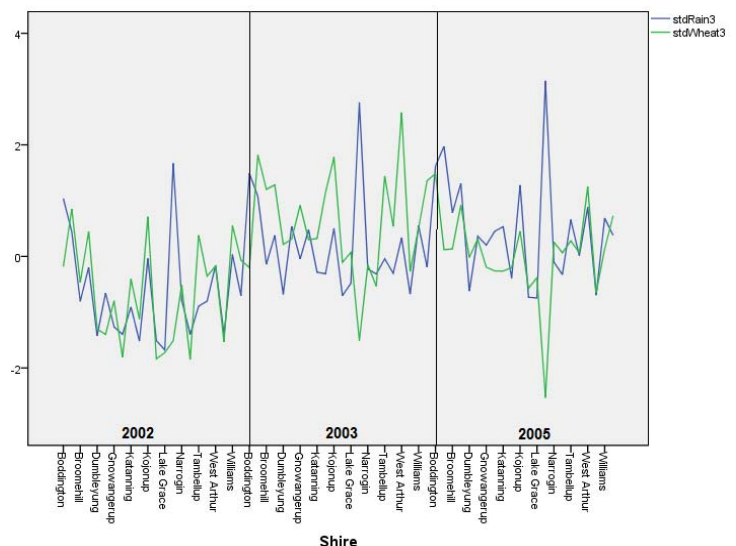

Fig. 4. Seq. plot of the std rainfall/wheat for $02,03 \& 05$

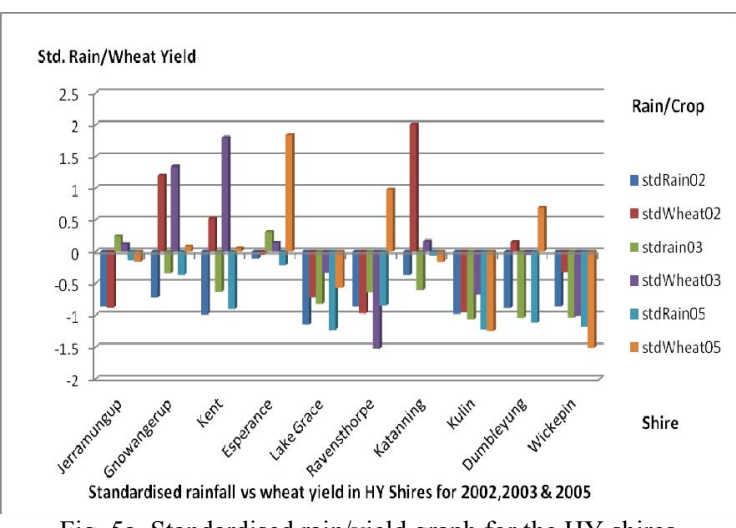

Fig. 5a. Standardised rain/yield graph for the HY shires 


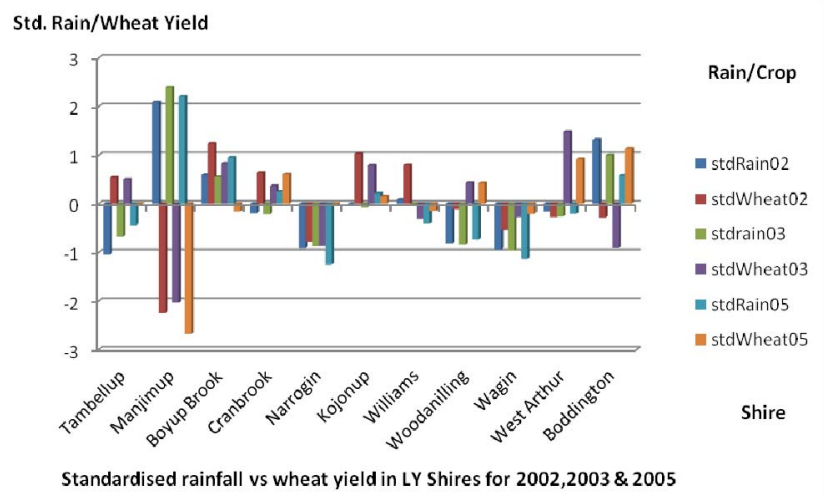

Fig. 5b. Standardised rain/yield graph for the LY shires

Fig. 5a and Fig 5b showed the standardised wheat crop yield and rainfall graph for the HY and LY shires respectively. Most of the HY shires in Figure 5a showed a positive correlation between rainfall and wheat yield, thereby demonstrating that a decrease in rainfall generally resulted in a decrease in wheat yield. There were some exceptions to this trend such as the shires of Gnowangerup and Kent which showed negative correlations for the year 2002 and 2003 and the shire of Katanning showing a negative correlation for the year 2002. The shires of Esperance, Ravensthorpe and Dumbleyung showed negative correlations for the year 2005. All of these exceptions resulted in high wheat yields from low rainfall. With reference to the LY shires in Figure 5b, the shire of Manjimup was considered to be an outlier due to unknown delivery area tonnage and wheat yield. The two LY shires of Narrogin and Wagin showed positive correlations with low rainfall matching the low wheat crop yields. The shire of Tambellup and Woodanilling showed a negative correlation but with a high wheat crop yield from low rainfall especially for the years 2002 and 2003. The overall trend for the LY shires was a high yield from low rainfall precipation. These trends were evident in Fig. $5 \mathrm{~b}$.

\section{E. DM analysis of the Wheat Crop Yields}

The next step in the individual scrutiny of the exercise was the use of regression in order to determine if the relationship established through correlation could be supported by a mechanism of predicting the wheat crop yield through the rainfall. This was carried out using the classification technique of data mining (DM) in the Waikato Environment for Knowledge Analysis (WEKA) software. The aggregated data for average annual rainfall and wheat crop yield for the 22 shires were used for this activity. The aggregated wheat crop yield and rainfall dataset was split up into a training set $(2001,2002,2004$, 2006 data) and a test set (2003 \& 2005 data). The exploratory part of the DM activity was to use the training set to determine the best-fit algorithm using a simple model of crop yield as a function of the location class and the average annual rainfall. All of the classification algorithms within WEKA were tested in this step and a short-list of six algorithms was selected. These algorithms were Gaussian Processes (GP), Multilayer Perceptron (MLP), Radial Based Function Network (RBF), Kstar, Sequential Minimal Optimisation (SMO) and Additive Regresssion (AR). All these algorithms use regression for predicting continous values in response to input values. GP is a form of regression where the distribution is over mean and covariance functions without hyper parameter tuning for the classifier function [22]; MLP is a feed forward multi-layer artificial neural network (ANN) function approximator classifier that uses the supervised learning technique of back propogation to classify instances [23]; RBF network is a neural network model function used for pattern classification [24]; SMO uses the support vector machine for its regression by quadratically scaling the number of training patterns [25]; the lazy Kstar algorithm is an instance based classifier that classifies a test instance based on its similarity to the training instance and $\mathrm{AR}$ is a meta classifier that seeks to enhance the performance of the regression based classifier [26]. Each of the algorithms trialled had different characteristics, correlation co-efficients and Root Mean Square Errors (RMSE) as shown in Table I.

\begin{tabular}{|c|c|c|c|c|}
\hline Weka Algorithm & $\begin{array}{c}\text { Correl } \\
\text { CoEff } \\
\text { Training }\end{array}$ & $\begin{array}{c}\text { RMSE } \\
\text { Training } \\
\text { Set }\end{array}$ & $\begin{array}{l}\text { RMSE } \\
\text { Cross } \\
\text { Valid } \\
\end{array}$ & $\begin{array}{c}\text { RMSE } \\
\text { Test } \\
\text { Set } \\
\end{array}$ \\
\hline Gaussian Processes & 0.998 & 0.411 & 0.718 & 1.057 \\
\hline MLP & 0.999 & 0.074 & 0.628 & 0.952 \\
\hline RBF Network & 0.754 & 0.549 & 0.619 & 0.794 \\
\hline SMOreg & 1.000 & 0.003 & 0.659 & 0.897 \\
\hline Kstar & 0.998 & 0.059 & 0.474 & 0.735 \\
\hline Additive Regression & 0.987 & 0.141 & 0.529 & 0.749 \\
\hline
\end{tabular}

TABLE II: WHEAT YIELD WEKA GP RESULTS MAXIMUM TEMPERATURE

\begin{tabular}{|c|c|c|c|c|c|c|}
\hline \multicolumn{4}{|c|}{$\begin{array}{l}\text { HIGH YIELD YEAR } \\
2003\end{array}$} & \multicolumn{3}{|c|}{$\begin{array}{l}\text { HIGH RAINFALL } \\
\text { YEAR } 2005\end{array}$} \\
\hline $\begin{array}{c}\text { Rural Shire } \\
\text { HY+ LY }\end{array}$ & $\begin{array}{l}\text { Actual } \\
\text { Yield } \\
\text { ton/ha }\end{array}$ & $\begin{array}{l}\text { Pred. } \\
\text { Yield } \\
\text { ton/ha }\end{array}$ & $\begin{array}{l}\% \\
\text { Error }\end{array}$ & $\begin{array}{c}\text { Actua } \\
1 \\
\text { Yield } \\
\text { ton/ha }\end{array}$ & $\begin{array}{c}\text { Pred. } \\
\text { Yield } \\
\text { ton } / \mathrm{h} \\
\mathrm{a} \\
\end{array}$ & $\begin{array}{l}\% \\
\text { Error }\end{array}$ \\
\hline Dumbleyung & 2.69 & 2.12 & 21.30 & 2.46 & 2.15 & 12.80 \\
\hline Esperance & 2.78 & 2.25 & 18.92 & 2.68 & 2.14 & 20.07 \\
\hline Gnowangerup & 3.37 & 2.07 & 38.55 & 2.29 & 2.14 & 6.64 \\
\hline Jerramungup & 2.77 & 2.01 & 27.47 & 2.22 & 2.14 & 3.78 \\
\hline Katanning & 2.79 & 2.07 & 25.91 & 2.22 & 2.15 & 3.29 \\
\hline Kent & 3.6 & 2.14 & 40.64 & 2.28 & 2.14 & 6.05 \\
\hline Kulin & 2.37 & 2.33 & 1.77 & 1.92 & 2.14 & 11.25 \\
\hline Lake Grace & 2.55 & 2.23 & 12.63 & 2.11 & 2.12 & 0.28 \\
\hline Ravensthorpe & 1.96 & 2.42 & 23.37 & 2.54 & 2.13 & 15.98 \\
\hline Wickepin & 2.21 & 2.21 & 0.09 & 1.84 & 2.15 & 17.07 \\
\hline Boddington & 2.28 & 2.28 & 0.13 & 3.93 & 2.19 & 44.38 \\
\hline Boyup Brook & 4.26 & 2.79 & 34.55 & 2.6 & 2.19 & 15.65 \\
\hline Broomehill & 3.65 & 2.24 & 38.63 & 2.61 & 2.14 & 17.89 \\
\hline Cranbrook & 3.73 & 2.12 & 43.14 & 3.38 & 2.15 & 36.39 \\
\hline Kojonup & 4.22 & 2.49 & 41.07 & 2.92 & 2.17 & 25.65 \\
\hline Narrogin & 2.33 & 2.33 & 0.13 & 2.22 & 2.19 & 1.44 \\
\hline Tambellup & 3.89 & 2.18 & 44.04 & 2.75 & 2.14 & 22.04 \\
\hline Wagin & 3 & 2.26 & 24.80 & 2.54 & 2.16 & 14.80 \\
\hline West Arthur & 5 & 2.42 & 51.56 & 3.7 & 2.19 & 40.73 \\
\hline Williams & 2.97 & 2.62 & 11.95 & 2.61 & 2.21 & 15.33 \\
\hline Woodanilling & 3.8 & 2.38 & 37.26 & 3.19 & 2.20 & 30.97 \\
\hline
\end{tabular}

GP, MLP and SMO were ruled out in the first instance due to the high RMSEs for the cross validation results. The GP algorithm had the second lowest RMSE for the predictions on the test data of 0.75 and a correlation co-efficient of 0.99 . Based on these results from Table I, together with a good cross validation result of 0.53 , the GP algorithm was selected and run for the prediction phase of the DM activity. The prediction results for years 2003 and 2005 were displayed side by side in Table II.

The shires in both tables were split up into HY (unshaded) and LY (shaded) shires. Good predictions were considered to have a percentage error of less than $20 \%$, average predictions 
a percentage error of $21-40 \%$ and weak predictions a percentage error of over $40 \%$. Accordingly, the HY shires of Esperance, Kulin, Lake Grace and Wickepin all had good predictions. The remaining HY shires of Dumbleyung, Jerramungup, Katanning, Gnowangerup and Ravensthorpe had average predictions and Kent a weak prediction. The LY shires of Boddington, Narrogin and Williams had good predictions while Boyup Brook, Broomehill, Wagin and Woodanilling had average predictions with weak predictions for Cranbrook, Tambellup, Kojonup and West Arthur.

Analysis of the prediction results for 2005 in Table II were again used to determine the accuracy of the predictions. Overall there were more positive prediction errors for the year 2005. On the good, average and low scale, the HY shires of Dumbleyung, Gnowangerup, Lake Grace, Jerramungup, Kent, Katanning, Kulin, Ravensthorpe and Wickepin had good predictions whilst Esperance only had an average prediction. There were no weak predictions for 2005. On the other hand, the LY shires of Boyup Brook, Broomehill, Narrogin, Williams and Wagin all had good predictions, with average predictions for Cranbrook, Kojonup, Tambellup and Woodanilling, and weak predictions for Boddington and West Arthur. Overall, the prediction errors were better in 2005 than in 2003 as depicted in Table II indicating the link to the rainfall where the wheat yield and predictions fell and rose slightly respectively as the rainfall increased.

\section{DISCUSSION}

In establishing a relationship between stochastic average annual rainfall and crop yield, a number of considerations had to be made such as: was rainfall a crucial factor in determining the final crop yield; whether rainfall was affected by the physical location in terms of elevation and other climatic conditions such as wind and temperature; the geographic and climatic scaling and resolution; the effect of using interpolated rainfall and the use of the shire crop yield in tonnes/hectare. Notwithstanding these effects and interactions, a pre-cursory relationship using a simple crop model (CM) was used where the classification entity was the rural shire and the average annual rainfall was used as a predictor. The production years of 2002, 2003 and 2005 were sampled as these were the years showing a considerable variation in both the attributes of rainfall and wheat yield. The selected study area had a number of intrinsic anomalies, such as shires within the study area that were not designated for cropping and cereal production land uses as well as outlier crop yield shires. The analysis was based on the earlier established metrics and on the general two stage of macroscopic and microscopic scrutiny. The macroscopic analysis involved the rainfall inspection and the individual wheat crop over the selected three years examinations, as part of an exploratory data mining (EDM) process. The simple bar graph visualization allowed the initial physical recognition of patterns and trends of wheat production as related to the stochastic average annual rainfall. The microscopic analysis involved scrutinizing the selected wheat crop yield for the selected years using DM. The results from the two stage analysis showed that there was some correlation between stochastic average annual rainfall and wheat yield. The sequence chart of the standardized rainfall and wheat yield was useful in showing a gradual shift in rainfall and crop yield across the HY shires. The results did show a high positive correlation between stochastic rainfall and the wheat yield, but there was a considerable amount of error in the predicted wheat yields which created uncertainty for the outcomes.

\section{CONCLUSION}

The actual wheat yields showed considerable variation between the 22 selected shires which contributed to the uncertainty of the prediction. As a consequence, it was concluded that rainfall may therefore not be such a decisive factor for wheat yield especially for the LY shires. The use of the data mining classification function of GP showed that the correlation between the stochastic average annual rainfall and wheat yield was a strongly positive one and that as a result generally, wheat yield in the South West agricultural region can be expected to increase with an increase in rainfall, but there could be an increasing under-estimation error in predicting the wheat yields. The uncertainty of the prediction was thought to be related to the influence of other factors such as the total seasonal rainfall as opposed to the rainfall for the months separately, as well as to the sparseness related to the intrinsic shire yield measurement of the dataset. It is thought that the performance of the WEKA algorithms could be enhanced by increasing the sample size of the crop yields from the three year selection to the 10 year selection for the years 2001-2010. Further investigation may also be needed in order to investigate the other elements of climate such as temperature as well as soil substrate and soil moisture respective to the area location under scrutiny. In addition, the other years within the 10 year series between 2001-2010 could be used for comparison with the three year selection of dry (2002), wet (2005) and productive (2003).

\section{ACKNOWLEDGMENTS}

The author wishes to especially acknowledge $\mathrm{Mr}$. Phil Goulding from the DAFWA organisation for his invaluable assistance in providing the data as well as his advice and suggestions on the sampling of the data.

\section{REFERENCES}

[1] J. R. Andersen, "Impacts of climatic variability on Australian agriculture: a review," Marketing and Agricultural Economics, vol. 47, pp. 147-177, 1979.

[2] R. Campbell, et al, "Impact of drought on national economy and employment," Quarterly Review of the Rural Economy, vol. 5, pp. 254-7, 1983

[3] P. Cantelaub and J. M. Terres, "Seasonal weather forecasts for crop yield modelling in Europe," Tellus A, pp. 476-487, 2004.

[4] D. B. Lobell and C. B. Field, "Global scale climate crop yield relationships and the impacts of recent warming," Environ. Res. Lett, vol. 2, 2007.

[5] E. M. Rasmassun and J. M. Wallace, "Meterological aspects of the El Nino/Southern Oscillation," Science, vol. 222, pp. 1195-202, 1983.

[6] A. B. Pittock, On the reality, stability, and usefulness of southern hemisphere teleconnections. Australian Meterological, pp. 75-82, 1984.

[7] N. Nicholls, Towards the prediction of major Australian droughts. Australian Meterological Magazine, pp. 161-166, 1985. 
[8] A. J. Challinor et al., "Toward a Combined Seasonal Weather and Crop Productivity Forecasting System: Determination of the Working Spatial Scale," ed, 2003.

[9] D. A. Atwood, "Aggregate food-supply and famine early warning.," Food Policy, vol. 16, pp. 245-251, 1991

[10] M. B. Smith and S. Davies, "Famine Early Warning and Response:The Missing Link," Intermediate Technology Publications, pp. 320, 1995

[11] H. Eakin, "Seasonal climate forecasting and the relevance of local knowledge," Physical Geography, vol. 20, pp. 447-460, 1999.

[12] B. Parthasarathy, et al, "Forecast of rainy-season foodgrain production based on monsoon rainfall," Indian Journal of Agricultural Science, vol. 62, pp. 1-8, 1992.

[13] M. A. Semanov and J. R. Porter, "Climatic variability and the modelling of crop yields," Agriculture for Meteorology, vol. 73, pp. 265-283, 1995.

[14] L. Mearns, et al, "Mean and variance change in climate scenarios: Methods, agricultural applications, and measures of uncertainty," Climatic Change, vol. 35, pp. 367-396, 1997.

[15] T. R. Wheeler, et al, "Temperature variability and the annual yield of crops," Agricultural Ecosystem Environment, vol. 82, pp. 159-167, 2000.

[16] A. V. M. Ines and J. W. Hansen "Bias correction of daily GCM rainfall for crop simulation studies," Agricultural and Forest Meteorology, vol. 138, pp. 44-53, 2006.
[17] R. J. Brooks, et al., "Simplifying Sirus: Sensitivity analysis and development of a meta-model for wheat yield prediction," European Journal of Agronomy, vol. 14, pp. 43-60, 2001.

[18] R. V. Martin, et al., "Seasonal maize forecasting for South Africa and Zimbabwe derived from an agroclimatological model," Journal of Applicable Meteorology, vol. 39, pp. 1473-1479, 2000.

[19] B. Basso, et al., "Spatial validation of crop models for precision agriculture," Agric. Syst., vol. 68, pp. 97-112, 2001.

[20] M. V. K. Sivakumar, "Climate prediction and agriculture: current status and future challenges," Climate Research, vol. 33, pp. 3-17, 2006.

[21] R. J. Hijmans, et al, "Very High Resolution Interpolated Climate Surfaces for Global Land Areas," International Journal of Climatology, vol. 25, pp. 1965-1978, 2005.

[22] C. E. Rasmussen, "Gaussian Processes in Machine Learning," Springer-Verlag Berlin, vol. 2004, pp. 63-71, 2004.

[23] J. M. Nazzal et al., "Multilayer Perceptron Neural Network (MLPs) For Analyzing the Properties of Jordan Oil Shale," World Applied Sciences, vol. 5, pp. 546-552, 2008

[24] S. Kung et al., Biometric Authentication: A Machine Learning Approach, NJ: Prentice Hall Press 2004.

[25] L. J. Cao et al., "Parallel Sequential Minimal Optimization for the Training of Support Vector Machines," IEEE Transactions ON Neural Networks, vol. 17, pp. 1039-49, 2006.

[26] I. H. Witten et al., Data Mining: Practical Machine Learning Tools and Techniques, 2011 